UDK 78:81'373.46

Prethodno priopćenje

Rukopis primljen 14. VII. 2018.

Prihvaćen za tisak 25. XI. 2018.

Paola Granado

Université Lumière Lyon 2

paola.granado@univ-lyon2.fr

Roger Miller

rmiller@numericable.com

\title{
SOME EXAMPLES FROM A CORPUS INVESTIGATION OF POTENTIALLY CONFUSING POLYSEMY AND FALSE FRIENDS IN THE TERMINOLOGY OF MUSIC, CENTRED ON ENGLISH AND FRENCH, WITH EQUIVALENTS IN FIVE OTHER LANGUAGES
}

This paper presents five of the initial items of a semantic study of confusable terms and false friends present in the lexis of music in English and French. All forms are also given in Italian, German, Spanish, Russian and Chinese. The object of study is a series of texts from corpora provided by New Sketch Engine, a product of Lexical Computing Limited. In the commentary section, the semantic variance between the different items within the same topic is studied. The approach is essentially synchronic, with priority being given to the examination of current usage. Diachronic considerations are also included where it has been possible to explain, or at least hypothesize how the meaning of the various terms has evolved. The main practical application of the paper is to provide a tool for reducing ambiguity in the area of music terminology. It will be of potential interest to all those concerned by the study and practice of music and the language of music in countries where the languages referred to in this study are in use.

\section{Objectives and methodology}

This research aims to reduce ambiguity and thus improve comprehension by exploring a selection of forty or so false friends between English and French in the area of music terminology. Translations into Spanish, Italian, German, Rus- 
sian and Chinese have been included to ensure that the research will be easily accessible to a vast number of linguists and musicians. With a total number of 152 terms explored, the research can be said to constitute a mini-corpus in its own right. The corpus consists of forty-two topics, each of which includes a certain number of related forms. The topics are presented in three sections, namely instruments, players and voices (1), notation (2) and performance (3). This division corresponds roughly to the areas of lexis to which the terms belong. The relationship between the forms within the same topic is analysed in the samples and commentaries section.

The main table (Figure 1) presents the English and French forms and their translation into five other languages. Cyrillic transliteration is provided for Russian and pinyin script, using Western characters, is used for Chinese.

The corpora examples (Figures 3 -9) are taken from eight of the corpora provided by New Sketch Engine. In the case of Russian (Figure 8) and Chinese (Figure 9), translations into English are given, but it was not deemed necessary to provide assistance of this kind for the Spanish, Italian and German references. As the Internet is in a constant state of flux, some of the websites used as sources in the corpora are no longer accessible. This is particularly so in the case of the Chinese corpus. They are identified as such by the ${ }^{\circ}$ symbol. In a small number of cases it proved impossible to provide examples from the corpora. The problem was solved by using Internet examples not quoted in the corpora. Unlike some of the corpora sources, they are, however, all currently accessible. The crawl dates indicating when the information was downloaded from the Internet indicate clearly which references are taken from the corpora as they are no later than 2013. The references added by the researchers themselves were all downloaded in 2018.

Between the main table and the corpora references there is a list of the English and French terms highlighting the false friends (Figure 2), which are identified by the letters $[\mathrm{BH}]$, standing for 'Bilingual Homophones' (cf. Reference to Knospe et al. 2016 in the following section). The extent to which the homophony generates confusion is clearly dependent on the language skills of the user. The bilingual user will have little or no difficulty in perceiving that homonymy is present, whereas the user with limited knowledge of the L2 may well experience confusion. There is a continuum between these two conditions. 
Owing to the required length of this paper, only the first / first two topics in each of the three sections are presented.

\section{The notion of false friends}

It is now important to look briefly at the notion of false friends, a term coined, in French as faux amis by Koessler and Derocquigny in 1928. A standard dictionary indicates that a false friend, also known as a false cognate, is "a word in a foreign language that seems similar to one in your own, so that you wrongly think they both mean the same thing" (Summers 1995).

Knospe, Onysko and Goth (2016) used the term bilingual homophones with regard to Finnish and Estonian, an association of languages where the phenomenon is particularly widespread. ${ }^{1}$

The homophones considered here are often associated with polysemy, which, as Stephen Ullman pointed out, is "the pivot of semantic analysis" (Ullman 1951). When seemingly similar terms exist in different languages, it is necessary to consider the range of meaning, and/or the acception of polysemes. This typically implies the existence of a more restricted meaning in one language of a term whose meaning in the other is more general, a phenomenon which is highlighted in our research by the use of the words general (gen.) and musical (mus.) to indicate the acception of the term concerned.

Here again, the awareness by L1 users of the existence of L2 polysemy naturally depends on their level of competence in the L2.

\section{Sample Topics and Commentaries}

\subsection{Item 1.01 Alto}

In both French and English, alto means a voice or instrument which is relatively low for female voices and high-pitched instruments such as the flute, and rela-

\footnotetext{
The languages [Finnish and Estonian] share a large amount of bilingual homophones, so-called false friends, words that sound similar but differ in meaning.
} 
tively high for male voices and medium or low-pitched instruments such as the trombone.

The false friends are alto in French, when it means the instrument belonging to the violin family which is usually called the viola in English, and alto in English, which usually refers to the voice. The polysemy that exists in French is absent in English.

The alto viol in English is, however, an instrument belonging not to the violin but to the viol family, all the members of which are played vertically. Interestingly enough, the American instrument-maker Carleen Hutchins designed and built a vertical viola in the 1960 s which she called an alto violin. ${ }^{2}$

Although alto was and is an Italian word meaning 'high', modern Italian nevertheless uses viola for the modern instrument belonging to the violin family. Spanish follows Italian, with viola, but Russian follows the French with альт ('al't'). Chinese has 中提琴 ('zhōng tí qín') meaning 'medium string instrument': half way between the violin (小提琴 'xiăotíqín' - small string instrument) and the cello (大提琴 'dà tí qín' - large string instrument). The German term is Bratsche, which derives from the Italian term viola da braccio 'arm viola', indicating that the instrument was and is normally played horizontally and rests on the arm, unlike the viola de gamba, which is played between the legs like the modern cello. Similar forms exist in Danish (bratch) and Hungarian (brácsa), although Hungarian also has mélyhegedü 'deep violin'.

Even in a monolingual French context there is a risk of ambiguity between the alto, meaning the voice, which the Grand Robert dictionary indicates was first attested as a noun in 1771 (Robert 1950), and alto meaning the instrument, which did not enter the language until 1808. This ambiguity is sometimes avoided by differentiating between the plural forms. The Italian form alti is often substituted for the standard French plural form altos for voices or singers, ${ }^{3}$ whereas altos is more usual for the instruments or their players. ${ }^{4}$

\footnotetext{
https://en.wikipedia.org/wiki/Vertical_viola.

Of the first 200 samples containing alti in the New Sketch Engine Corpus, 20 refer to voices and only 11 to instruments

4 Of the first 200 samples containing altos in the corpus, 86 refer to instruments and only 45 to voices
} 


\subsection{Item 1.02 Bar}

A bar is the thin strip of metal or wood of a xylophone, marimba, vibraphone etc. which is struck with a mallet to create the sound. The term is derived from the shape of the object and does not mean a division or separation. The same word is, of course, also used in English to denote the long counter where drinks are served and, by extension, the room or establishment where it is situated. This is the only meaning of bar in French, other than the polyseme meaning 'barfish' or 'sea bass'. French uses lame for the musical sense of 'bar'. Italian with lamella, and Spanish with lamina, both use related forms.

Although there is little or no risk of misunderstanding, this is nevertheless an example of false friends. The Grand Robert indicates that bar entered French from English in the mid nineteenth century, but only in the "general" sense, thus creating a term whose contextual acceptance is, paradoxically, more restricted, in that the musical application is excluded.

\subsection{Item 2.01 Bar}

The term bar also appears in the "notation" section of the study. Here the false friends occur because the French word barre (usually coupled with mesure in the term barre de mesure) is bar-line in British English and bar in English is mesure in French. American English uses measure and not bar so the notion of false friends does not apply.

Bar came into English from Norman French barre with the meaning of a barrier or separation. ${ }^{5}$ It still had this meaning at the end of the nineteenth century, as is attested by the following entry in the Pronouncing Musical Dictionary by $\mathrm{H}$. A. Clarke (1896):

Bar. A line drawn across the staff or staves to divide the music into portions of equal duration. The portion enclosed between two bars is called a measure. The almost universal custom of musicians, however, is to use bar in the sense of measure.

\footnotetext{
https://www.etymonline.com/word/bar
} 
The use of bar to mean a segment of music is therefore a metonymy from the original usage: the action of dividing came to mean the result of this action. According to Sablayrolles (2010), a phenomenon of this type constitutes a shift of meaning rather than a neologism as such. The original sense survives, in the term double bar, which is not two bars, as might be supposed, but two bar-lines.

Forty years later however, it is apparent from the entry in the Oxford Companion to Music by Scholes (1938) that the Americans had rejected the new usage, which had, on the contrary, been adopted by the British:

Measure. [...] The time-space of a group, i.e. between one strong accent and another, is called, in the terminology of older British writers, in that of John Curwen and his followers [...] and in that of all modern American writers, a measure.

The measures are, in notation, marked off from one-another by 'bars' or 'bar lines' before each of the strongly accented beats. As a consequence, the British have come to use the word bar for 'measure', which is illogical and yet seems to cause little confusion.

\subsection{Item 2.02 ' $C$ '}

The name given to the note ' $\mathrm{C}$ ' is problematical for two reasons, one of them derived from the difference between the phonological systems of French and English and the other from the different systems used for naming the notes.

To linguists and skilled users of French and English, the risk of confusing English $C$ and French si may seem minimal because the phonetic supra-segmental differences with respect to (lip) tension and length are substantial: $C$ is long and without lip tension, $s i$ is short and tense. Inexperienced L1 language users will naturally impose their own phonological usage on the L2 term, thus creating ambiguity. This phenomenon of L1 interference is well-known.

The alphabetical system of notation $(\mathrm{A}, \mathrm{B}, \mathrm{C})$ is not necessarily familiar to French-speakers and the seventh degree of the 'do, re, mi' system may not be known to English speakers. The latter problem arises from the fact that although the solmization (do, re, mi) system is used in music education throughout the English-speaking world, the seventh degree is not 'si', but ' $\mathrm{i}$ '. The change to the 
original 'si' was introduced in the nineteenth century by Sarah Glover and John Curwen in order to attribute a different initial consonant to each note, 's' being already employed for the fifth degree. ${ }^{6}$

The original term for the first degree in the solmization system invented by Guido d'Arezzo in the eleventh century was ut. 'Do' was substituted in the seventeenth century because it facilitated the production of acceptable vocal tone when solmizing (singing with the names of the notes for learning purposes). $U t$ survives in French as an alternative still used for the pitch of instruments (trompette en $u t$ ), high notes (contre $u t$ ) and the title of certain works, notably the Symphonie en ut by Bizet.

A further problem of ambiguity is to be found in the use of the letters B and $\mathrm{H}$ in a number of countries including Germany and Croatia for the two notes known elsewhere as B flat and B (natural) respectively.

\subsection{Item 3.01 Dynamics}

The inclusion in this study of the term dynamics, used in English to describe the basic notion of the volume required in performance, ${ }^{7}$ is somewhat controversial because it can only be considered a false friend with dynamiques if we accept the hypothesis that French musicians prefer to use the term nuance to convey this meaning. ${ }^{8}$ It is certainly true that dynamique can also used in the sense of the intensity of a sound, ${ }^{9}$ but in the authors' experience, this term would appear to be more frequently used by musicologists than by musicians. A nuance in English is of course 'a very slight, hardly distinguishable difference in manner, colour, meaning, etc.' (Summers 1995). Both dynamics and dynamique have several meanings, including, for example, in French, the 'totality of the forces in interaction or in opposition in a phenomenon or a structure'. ${ }^{10}$ Both for nuances and dynamics then, the acception is restricted in the musical context.

\footnotetext{
See Dumorest (2003)

"That part of musical expression concerned with the varying degrees of intensity (loudness) of the sound produced” (Scholes 1938).

8 "Degré divers de douceur ou de force à donner aux sons"(Robert 1950).

9 "Utilisation de différentes intensités sonores" (Siron 2002).

10 "Ensemble des forces en interaction et en opposition dans un phénomène, une structure" (Robert 1950).
} 


\section{Conclusion}

In conclusion it can be observed that there is no cross-linguistic standardisation of the boundaries between general and musical usage. Furthermore, it is often the difference between the two that is responsible for the ambiguity created by the existence of bilingual homophones.

This research is ongoing, and commentaries will eventually be added to all the topics in the corpus. Although all the terms have been translated into five languages other than French and English, it has not yet been possible to add critical comments on the presence or absence of false friends. It would clearly be of interest at a future date to study the occurrence of false friends in the context of other combinations of languages. Another area that remains to be explored is the occurrence of neologisms, notably in the light of recent research by JeanFrançois Sablayrolles (2010) and by John Humbley (2010).

Data

\begin{tabular}{|c|c|c|c|c|c|c|c|c|}
\hline \multicolumn{9}{|c|}{ Figure 1 - Main table } \\
\hline & Spanish & Ifalian & French & English & German & Russian & Russian & Chinese \\
\hline & & & & & & (cyrillic) & (transliteration) & (pinyin) \\
\hline \multicolumn{9}{|c|}{ Section 1 - Instruments, players and voices } \\
\hline & & & & & & & & \\
\hline $1.01 \mathrm{~A}$ & alto & alto & alto (voice) & alto (voice) & Alt(stimme) & aлbT & $\mathrm{al}^{\prime} \mathrm{t}$ & zhōng yīn \\
\hline $1.01 \mathrm{~B}$ & viola & viola & alto (instr.) & viola & Bratsche & aльт & al't & zhōng ti gin \\
\hline $1.01 \mathrm{C}$ & contralto & contralto & contralto & contralto & Kontralt & контральто & kontral'to & nü dì dìn \\
\hline $1.02 \mathrm{~A}$ & lamina & lamella & $\begin{array}{l}\text { lame (xylophone, } \\
\text { etc.) }\end{array}$ & $\begin{array}{l}\text { bar (xylophone, } \\
\text { etc.) }\end{array}$ & Klangstab & бpycs & brusk & qín jiàn \\
\hline $1.02 \mathrm{~B}$ & barra & bar & bar & bar (gen.) & Lokal & бap & bar & jiŭ bā \\
\hline \multicolumn{9}{|c|}{ Section 2 - Notation } \\
\hline 2014 & comnás & & mesure & & Takt & TаKT & takt & viăoiié \\
\hline$\frac{2.01 \mathrm{~A}}{2.01 \mathrm{~B}}$ & $\begin{array}{l}\text { compas } \\
\text { compás }\end{array}$ & $\begin{array}{l}\text { battuta } \\
\text { battuta }\end{array}$ & mesure & \begin{tabular}{|l|} 
bar (UK) \\
measure (US)
\end{tabular} & Takt & TakT & $\begin{array}{l}\text { takt } \\
\text { takt }\end{array}$ & $\begin{array}{l}\text { Maoje } \\
\text { xiăojié }\end{array}$ \\
\hline $2.01 \mathrm{C}$ & barra de compás & barretta & barre de mesure & bar-line & Taktstrich & tákтовая черта́ & táktovaja čertá & xiǎojié xiàn \\
\hline $2.01 \mathrm{D}$ & doble barra & $\begin{array}{l}\text { doppia } \\
\text { stanghetta }\end{array}$ & double barre & double-bar & Doppelstrich & Двойна́я та́ксовая & $\begin{array}{l}\text { dyoináia táktovaja } \\
\text { čertá }\end{array}$ & $\begin{array}{l}\text { shuāng xiăo } \\
\text { jié xiàn }\end{array}$ \\
\hline $2.02 \mathrm{~A}$ & do & do & do & C & C & до & do & C \\
\hline $2.02 \mathrm{~B}$ & do & do & ut & C & C & до & do & C \\
\hline $2.02 \mathrm{C}$ & si & si & si & B & $\mathrm{H}$ & сй & si & B \\
\hline $2.02 \mathrm{D}$ & si bemol & si bemolle & si bémol & B flat & B & си-бемоль & si-bemol' & jiàng $B$ yin \\
\hline \multicolumn{9}{|c|}{ Section 3 - Performance } \\
\hline $3.01 \mathrm{~A}$ & dinámica & dinamiche & nuances (mus.) & dynamics (mus.) & Nuance & Toнкость & tonkost' & lìdù \\
\hline $3.01 \mathrm{~B}$ & dinámica & dinamico & dynamique (gen.) & dynamic (gen.) & Dynamik & динамика & dinamika & $\begin{array}{l}\text { shêng gì bó } \\
\text { bố }\end{array}$ \\
\hline $3.01 \mathrm{C}$ & matiz & sfumatura & détail & nuance (gen.) & Einzelheit & HroaHC & nyuans & xì jié \\
\hline
\end{tabular}


P. Granado, R. Miller: Some examples from a corpus investigation...

\begin{tabular}{|l|l|l|}
\hline Figure 2 & Identification of false friends \\
\hline & French & English \\
\hline 1.01A & alto (voix) & alto (voice) $[\mathrm{BH}]$ \\
\hline $1.01 \mathrm{~B}$ & alto (instr.) $[\mathrm{BH}]$ & viola \\
\hline $1.01 \mathrm{C}$ & contralto & contralto \\
\hline 1.02A & lame & bar [BH] \\
\hline 1.02B & bar [BH] & bar (gen.) \\
\hline 2.01A & mesure & bar (UK) $[\mathrm{BH}]$ \\
\hline 2.01B & mesure & measure (US) \\
\hline 2.01C & barre de mesure $[\mathrm{BH}]$ & bar-line \\
\hline 2.01D & double barre & double-bar \\
\hline 2.02A & do & C [BH] \\
\hline 2.02B & ut & C \\
\hline 2.02C & si [BH] & B \\
\hline 2.02D & si bémol & B flat \\
\hline 3.01A & nuances (mus.) & dynamics (mus.) $[\mathrm{H}]$ \\
\hline 3.01B & dynamigue (gen.) $[\mathrm{H}]$ & dynamic (gen.) \\
\hline 3.01C & détail & nuance (gen.) \\
\hline [BH] bilingual homophones & \\
\hline & & \\
\hline
\end{tabular}

\begin{tabular}{|c|c|c|c|c|c|}
\hline \multicolumn{6}{|c|}{ Figure 3 French corpus references } \\
\hline Item $N^{\circ}$ & Preceding collocation & Item & Following collocation & URL & Crawl Date \\
\hline & & & & - source no longer accessible & \\
\hline \multicolumn{6}{|c|}{ Section 1 - Instruments, players and voices } \\
\hline $1.01 \mathrm{~A}$ & je chante des morceaux $\mathrm{d}^{\prime}$ & alto & $\begin{array}{l}\text { : de mezzo-soprano et de } \\
\text { soprano }\end{array}$ & http $/ /$ /vigneherve free fr/ & $2012-02-28$ \\
\hline $1.01 \mathrm{~B}$ & $\begin{array}{r}\text { trompette, trombone, } \\
\text { violon, }\end{array}$ & alto & - violoncelle batterie, harpe & $\begin{array}{l}\text { http } / / \text { atoryastatinachat awardspace biz/metro } \\
\text { nidazole-acheter html }\end{array}$ & $2012-02-27$ \\
\hline $1.01 \mathrm{C}$ & $\begin{array}{l}\text { elle est écrite pour deux } \\
\text { sopranos, deux }\end{array}$ & contraltos & $\begin{array}{l}\text {, un ténor, deux récitants, un } \\
\text { choeur de solistes }\end{array}$ & $\begin{array}{l}\text { http//mediaplan.ovh.net/-remue/spip.php? } \\
\text { article2438 }\end{array}$ & $2012-02-29$ \\
\hline $1.02 \mathrm{~A}$ & violoncelle, contrebasse, les & lames & $\begin{array}{l}\text { de xylophone et de marimba } \\
\text { également }\end{array}$ & $\begin{array}{l}\text { https } / / \text { /brindauvergne.com/contenu.php? } \\
\text { id_contenu }=80 \text { \&id_dossier }=35\end{array}$ & $2012-02-22$ \\
\hline $1.02 \mathrm{~B}$ & $\begin{array}{r}\text { à l'opposé du restaurant } \\
\text { Avenues, le }\end{array}$ & bar & $\begin{array}{l}\text { yous accueille avec ses } \\
\text { chaleureuses }\end{array}$ & $\begin{array}{l}\text { ohttps://www grandluxuryhotels com/tag/rev } \\
\text { iew }\end{array}$ & $2012-02-27$ \\
\hline \multicolumn{6}{|c|}{ Section 2-Notation } \\
\hline $2.01 \mathrm{~A}$ & $\begin{array}{r}\text { leurs pas. Si nous } \\
\text { numérotons les temps de la }\end{array}$ & mesure & $\begin{array}{l}\text { musicale "1", "2", "3" et "4", le } \\
\text { danseur de Mambo }\end{array}$ & $\begin{array}{l}\text { http } / / \text { www salsayirus.com/Qu-est-ce-que- } \\
\text { le-fameux-N-Y-Style.htm1 }\end{array}$ & $2012-02-28$ \\
\hline $2.01 \mathrm{~B}$ & $\begin{array}{r}\text { leurs pas. Si nous } \\
\text { numérotons les temps de la }\end{array}$ & mesure & $\begin{array}{l}\text { musicale "1", "2","3" et "4", le } \\
\text { danseur de Mambo }\end{array}$ & $\begin{array}{l}\text { http } / / \text { www salsayinus com/Qu-est-ce-que- } \\
\text { le-fameux-N-Y-Style.htm1 }\end{array}$ & $2012-02-28$ \\
\hline $2.01 \mathrm{C}$ & $\begin{array}{l}\text { 1a lecture de l'écriture } \\
\text { musicale. La double }\end{array}$ & barre & $\begin{array}{l}\text { de mesure signifie la fin d'un } \\
\text { morceau ou un }\end{array}$ & $\begin{array}{l}\text { http } / / \text { chaisemusicale be/petit-lexique-de- } \\
\text { solfege-al-1-usage-des-parents-1.php? } \\
\text { mode=edit }\end{array}$ & $2012-02-21$ \\
\hline 2.01D & $\begin{array}{l}\text { la lecture de l'écriture } \\
\text { musicale. La }\end{array}$ & double barre & $\begin{array}{l}\text { de mesure signifie la fin d'un } \\
\text { morceau ou un }\end{array}$ & $\begin{array}{l}\text { http } / / \text { chaisemusicale be/petit-lexique-de- } \\
\text { solfege-a-1-usage-des-parents-1.php? } \\
\text { mode=edit }\end{array}$ & 2012-02-21 \\
\hline
\end{tabular}




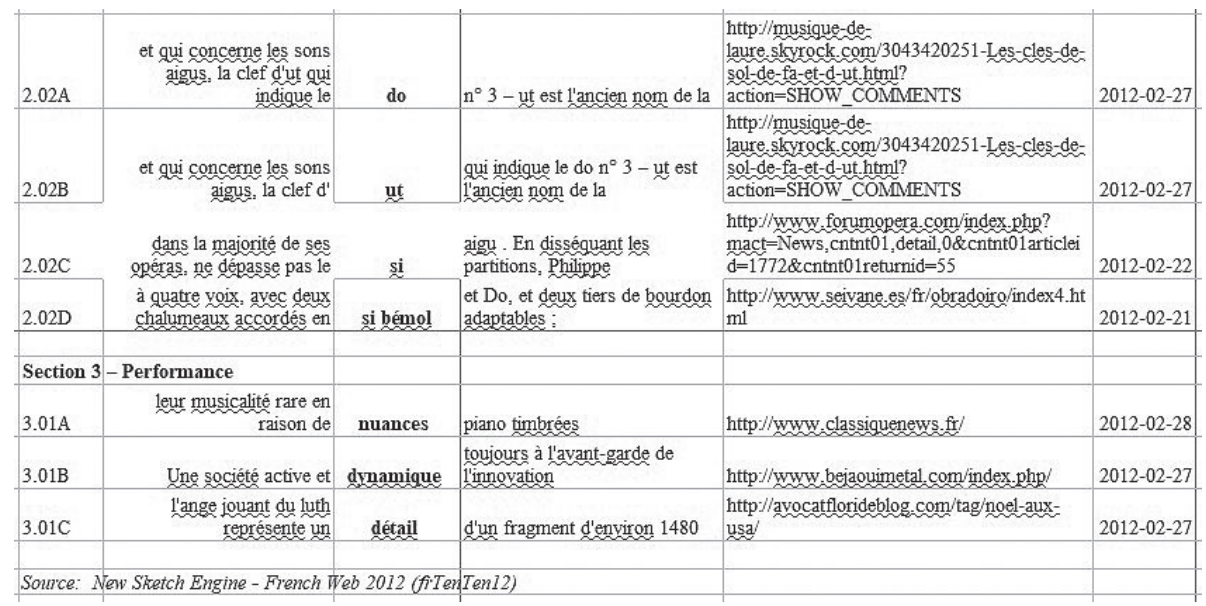

\begin{tabular}{|c|c|c|c|c|c|c|c|}
\hline Figure 4 & English corpus references & & & & & & \\
\hline \multirow[t]{2}{*}{ Item $N^{\circ}$} & Preceding collocation & Item & Following collocation & URL/title & Type & Author & Crawl Date \\
\hline & & & & - source no longer accessible & & & \\
\hline \multicolumn{8}{|c|}{ Section 1 - Instruments, players and voices } \\
\hline $1.01 \mathrm{~A}$ & $\begin{array}{r}\text { she turned her head in the } \\
\text { direction of the }\end{array}$ & alto & : a young woman with & Esquire & Periodical & & 1991 \\
\hline $1.01 \mathrm{~B}$ & $\begin{array}{l}\text { evidently too thin: he adds } \\
\text { second violins and }\end{array}$ & violas & , mainly on the off-bea & Early Music & Periodical & & 1993 \\
\hline $1.01 \mathrm{C}$ & $\begin{array}{l}\text { never seemed to make up } \\
\text { his mind whether I was a }\end{array}$ & contralto & or a soprano. & $\begin{array}{l}\text { Peter Grimes. Gloriana. } \\
\text { English National Opera Guide } \\
24\end{array}$ & Book & $\begin{array}{l}\text { Slater, Montagu, } \\
\text { Plomer, William \& } \\
\text { Crabbe, George }\end{array}$ & 1983 \\
\hline $1.02 \mathrm{~A}$ & $\begin{array}{r}\text { similar to a xylophone, } \\
\text { made with }\end{array}$ & rooden bars & $\begin{array}{l}\text { but with tubular metal } \\
\text { resonators fixed }\end{array}$ & $\begin{array}{l}\text { Orchestral technique: a } \\
\text { manual for students }\end{array}$ & Book & Jacob, Gordon & 1982 \\
\hline $1.02 \mathrm{~B}$ & if you walked into a & bar & in Babylon around 200 & Guinness & Miscellaneous & & $1985-1993$ \\
\hline
\end{tabular}

\begin{tabular}{|c|c|c|c|c|c|c|c|}
\hline \multicolumn{8}{|c|}{ Section 2-Notation } \\
\hline $2.01 \mathrm{~A}$ & $\begin{array}{l}\text { the second time through } \\
\text { you miss out the }\end{array}$ & rst time ba' & $\begin{array}{l}\text { second time bar (9) } \\
\text { and continue }\end{array}$ & Guitarist & \multicolumn{2}{|l|}{ Periodical } & $1985-1993$ \\
\hline $2.01 \mathrm{~B}$ & if you walked into a & bar & in Babylon around 200t & Guinness & \multicolumn{2}{|l|}{ Miscellaneous } & $1985-1993$ \\
\hline $2.01 \mathrm{C}$ & $\begin{array}{r}\text { of a bar-line stave (not } \\
\text { shown) ensures that }\end{array}$ & bar-lines & are placed automaticall & Practical PC & Periodical & & $1985-1993$ \\
\hline $2.01 \mathrm{D}$ & $\begin{array}{r}\text { separated in the manuscript } \\
\text { by a }\end{array}$ & double-bar & $\begin{array}{l}\text { conclusion, but calls } \\
\text { for a }\end{array}$ & Early Music & Periodical & & 1993 \\
\hline $2.02 \mathrm{~A}$ & $\begin{array}{l}\text { only a few weeks left to } \\
\text { live. He played Mozart's }\end{array}$ & C major & Concerto, K467 & Conversations with Karajan & Book & Osborne, Richard & 1991 \\
\hline $2.02 \mathrm{~B}$ & $\begin{array}{l}\text { only a few weeks left to } \\
\text { live. He played Mozart's }\end{array}$ & C major & Concerto, K467 & Conversations with Karajan & Book & Osborne, Richard & 1991 \\
\hline $2.02 \mathrm{C}$ & $\begin{array}{r}\text { a celebrated recording of } \\
\text { Bach's }\end{array}$ & B minor & $\begin{array}{l}\text { Mass. In its grace, } \\
\text { fire, and expressive }\end{array}$ & Conversations with Karajan & Book & Osborne, Richard & 1991 \\
\hline $2.02 \mathrm{D}$ & Peter slides up a semitone, & B flat & to C flat, on the words & $\begin{array}{l}\text { Peter Grimes. Gloriana. } \\
\text { English National Opera Guide } \\
24\end{array}$ & Book & $\begin{array}{l}\text { Slater, Montagu, } \\
\text { Plomer, William \& } \\
\text { Crabbe, George }\end{array}$ & 1983 \\
\hline \multicolumn{8}{|c|}{ Section 3 - Performance } \\
\hline $3.01 \mathrm{~A}$ & $\begin{array}{r}\text { an impressive start; the } \\
\text { terraced }\end{array}$ & dynamics & $\begin{array}{l}\text { and tempi of the } \\
\text { opening section }\end{array}$ & CD Review & Periodical & & 1992 \\
\hline $3.01 \mathrm{~B}$ & $\begin{array}{r}\text { Guinness operates in a } \\
\text { changing, }\end{array}$ & dynamic & $\begin{array}{l}\text { and competitive } \\
\text { environment }\end{array}$ & Guinness brewing worldwide & Miscellaneous & & $1985-1993$ \\
\hline $3.01 \mathrm{C}$ & $\begin{array}{r}\text { two or three pauses, and } \\
\text { every }\end{array}$ & nuance & $\begin{array}{l}\text { of verbal rhythm, as } \\
\text { well as pitch, is } \\
\text { notated }\end{array}$ & $\begin{array}{l}\text { Peter Grimes. Gloriana. } \\
\text { English National Opera Guide } \\
24\end{array}$ & Book & $\begin{array}{l}\text { Slater, Montagu, } \\
\text { Plomer, William \& } \\
\text { Crabbe, George }\end{array}$ & 1983 \\
\hline urce & retch Engine $-3.01 \mathrm{~A}$ and 3.0 & m Engh & b 2013 ( & & & & \\
\hline
\end{tabular}




\begin{tabular}{|c|c|c|c|c|c|c|c|c|c|}
\hline \multicolumn{10}{|c|}{ Figure 5 Spanish corpus references } \\
\hline \multirow[t]{2}{*}{ Item $N^{\circ}$} & Preceding collocation & \multicolumn{2}{|l|}{ Item } & \multicolumn{2}{|c|}{ Following collocation } & \multicolumn{2}{|l|}{ Website } & URL & \multirow{2}{*}{ Crawl Date } \\
\hline & & & & & & \multicolumn{3}{|c|}{ - source no longer accessible } & \\
\hline \multicolumn{10}{|c|}{ Section 1 - Instruments, players and voices } \\
\hline $1.01 \mathrm{~A}$ & $\begin{array}{r}\text { llegada del músico a } \\
\text { Leipzig. Fstấ escrita para } \\
\text { yoces de }\end{array}$ & \multicolumn{2}{|l|}{ alto } & \multicolumn{2}{|c|}{$\begin{array}{l}\text { y tenor, con coro, corno da } \\
\text { caccia, dos oboes, }\end{array}$} & \multicolumn{2}{|l|}{ femas es } & http//Www femas. es/notas_dia18-1.html & 2011 \\
\hline $1.01 \mathrm{~B}$ & $\begin{array}{r}\text { Un repertorio poco habitual, } \\
\text { el de la }\end{array}$ & \multicolumn{2}{|l|}{ viola } & \multicolumn{2}{|c|}{$\begin{array}{l}\text { de gamba concertante, es el } \\
\text { gue nos ofrece esta }\end{array}$} & \multicolumn{2}{|l|}{ elcultural.es } & $\begin{array}{l}\text { http//wwww elcultural.es/version_pape//MU } \\
\text { SICA/13233/Viola_da_gamba_concertata }\end{array}$ & 2011 \\
\hline $1.01 \mathrm{C}$ & $\begin{array}{r}\text { a seis voces dividida en } \\
\text { sopranos I y II, }\end{array}$ & \multicolumn{2}{|c|}{ contralto } & \multicolumn{2}{|c|}{$\begin{array}{l}\text { tenores I y II y baios, de } \\
\text { extrema densidad, }\end{array}$} & \multicolumn{2}{|l|}{ femas.es } & http $/ /$ www femas es/notas dia $20-3 . \mathrm{html}$ & 2011 \\
\hline $1.02 \mathrm{~A}$ & cada & \multicolumn{2}{|c|}{ lamina } & \multicolumn{2}{|c|}{$\begin{array}{l}\text { se afina segin un tono } \\
\text { especifico (nota musical) de } \\
\text { la escala cromática }\end{array}$} & & $\begin{array}{l}\text { https } / / \text { es. wikipedia.org/wiki/Xil } \\
\% \text { C3\%B3fono }\end{array}$ & 2018 \\
\hline $1.02 \mathrm{~B}$ & $\begin{array}{r}\text { ciudades que constan de } \\
\text { stands simulando }\end{array}$ & \multicolumn{2}{|l|}{ barra } & \multicolumn{2}{|c|}{$\begin{array}{l}\text { de bares donde los } \\
\text { estudiantes son retados a }\end{array}$} & \multicolumn{2}{|c|}{ marketingnews.es } & $\begin{array}{l}\text { http } / / \text { www marketingnews.es/gran- } \\
\text { consumo/noticia/1044664028005/axe- } \\
\text { anuncia-primera-yez-television-pack- } \\
\text { navideno.1.html }\end{array}$ & 2011 \\
\hline \multicolumn{10}{|c|}{ Section 2-Notation } \\
\hline $2.01 \mathrm{~A}$ & $\begin{array}{l}\text { que este año ha contado } \\
\text { con buen clima. Al }\end{array}$ & \multicolumn{2}{|c|}{ compás } & $\begin{array}{l}\text { la Banda de } \\
\text { participante }\end{array}$ & $\begin{array}{l}\text { Música, los } \\
\text { shan }\end{array}$ & salinas.es & & $\begin{array}{l}\text { http://www salinas.es/noticias/gran- } \\
\text { participacion-en-el-carnaval }\end{array}$ & 2011 \\
\hline $2.01 \mathrm{~B}$ & $\begin{array}{l}\text { termino que kiere decir } \\
\text { ocho versos. en }\end{array}$ & compá & & $\begin{array}{l}\text { de } 5 \text { partes } \\
\text { tamborily } y\end{array}$ & $\begin{array}{l}\text { acompañado por } \\
\text { histus }\end{array}$ & xuletas.es & & $\begin{array}{l}\text { http//wwww xuletas.es/directorio/chuletas/ } \\
\text { materia/musica/ordenar/fecha/ }\end{array}$ & 2011 \\
\hline $2.01 \mathrm{C}$ & on un largo preludio sin & barra de co & mpás & . que en ori & & femas.es & & http//www femas es/notas_dia $10 . h t m l$ & 2011 \\
\hline 2.01D & $\begin{array}{c}\text { el flamenco, y son cinco } \\
\text { notas conectadas por la }\end{array}$ & doble ba & & $\begin{array}{l}\text { Las division } \\
\text { seisillos, son }\end{array}$ & nes de seis, los & & & $\begin{array}{l}\text { http } / / \text { www cty es/USERS/norman/timec.h } \\
\text { tm }\end{array}$ & 2011 \\
\hline $2.02 \mathrm{~A}$ & $\begin{array}{l}\text { el jazz, el pop o el flamenco. } \\
\text { Incluso ha dado el }\end{array}$ & do & & $\begin{array}{l}\text { en algunas b } \\
\text { que ha realiz }\end{array}$ & $\begin{array}{l}\text { gandas sonoras } \\
\text { zado. }\end{array}$ & esmadrid.es & & $\begin{array}{l}\text { http } / / \text { www esmadrid. es/es/cargarBuscador } \\
\text { Agendado? } \\
\text { fechaDesde }=01 / 02 / 2011 \& \text { fechaHasta }=28 / 0 \\
2 / 2011 \& \text { codigoEvento=M18535\& codigoLo } \\
\text { cal=158\&tipo=2 }\end{array}$ & 2011 \\
\hline $2.02 \mathrm{~B}$ & $\begin{array}{l}\text { el jazz, el pop o el flamenco. } \\
\text { Incluso ha dado el }\end{array}$ & do & & $\begin{array}{l}\text { en alpunas b } \\
\text { que ha realiz }\end{array}$ & $\begin{array}{l}\text { gandas sonoras } \\
\text { zado: }\end{array}$ & esmadrid.es & & $\begin{array}{l}\text { http } / / \text { www esmadrid es/es/cargarBuscador } \\
\text { Agendado? } \\
\text { fechaDesde }=01 / 02 / 2011 \& \text { fechaHasta }=28 / 0 \\
2 / 2011 \& \text { codigoEvento=M18535\&codigoLo } \\
\text { cal=158\&tipo=2 }\end{array}$ & 2011 \\
\hline $2.02 \mathrm{C}$ & $\begin{array}{r}\text { de la tónica y resolviendo } \\
\text { con cromatización la }\end{array}$ & notas & & del registro & & riie.com.es & & http $/ /$ riie com.es $/ ? \mathrm{a}=39203$ & 2011 \\
\hline $2.02 \mathrm{D}$ & $\begin{array}{l}\text { y suena una octaya más } \\
\text { grave que el clarinete en }\end{array}$ & si bemo & & $\begin{array}{l}\text { En el jazz, } \\
\text { tenido un pa }\end{array}$ & $\begin{array}{l}\text { el clarinete ha } \\
\text { apel }\end{array}$ & mcues & & $\begin{array}{l}\text { http } / / \text { ocne.mcu es/temporada- } \\
\text { proyectoeducativo. asp? } \\
\text { l=es\&s=-materialdidactico }\end{array}$ & 2011 \\
\hline Section & 3-Performance & & & & & & & & \\
\hline $3.01 \mathrm{~A}$ & $\begin{array}{l}\text { Esto se ve, por ejemplo, en } \\
\text { las indicaciones de }\end{array}$ & dinámic & & en la partitur & & https //books.goo & oogle fr & $\begin{array}{l}\text { https } / / \text { books, google fr } / \text { books? } \\
\text { isbn }=8460961958\end{array}$ & 2011 \\
\hline $3.01 \mathrm{~B}$ & $\begin{array}{l}\text { sin ningun coste. Somos } \\
\text { una Empresa joven } y\end{array}$ & dinámic & & $\begin{array}{l}\text { de reciente } \\
\text { profesionale }\end{array}$ & creación por & acepto.es & & $\begin{array}{l}\text { http//www acepto_es/trabajo-de/reforma- } \\
\text { de-yivienda-caceres-RFF_1155 }\end{array}$ & 2011 \\
\hline $3.01 \mathrm{C}$ & $\begin{array}{l}\text { gusto y el tacto por los } \\
\text { materiales y colores, ell }\end{array}$ & matiz & & , la metáfora & a, el principio de & elcultural.es & & $\begin{array}{l}\text { http//Www _elcultural.es/version_papel/AR } \\
\text { TE/2527/Catherine_Lee }\end{array}$ & 2011 \\
\hline Source: $\mathrm{Ne}$ & ew Sketch Engine - Spanish Web 20 & 11 (esTenTen) & $1, \mathrm{Eu}+$ & $+\mathrm{Am})$ & & & & & \\
\hline Figure 6 & 5 Italian corpus references & & & & & & & & \\
\hline Item $N^{4}$ & Preceding & collocation & & Item & Following colloc & cation & URL & & Crawl Date \\
\hline & & & & & & & ${ }^{\circ}$ sourc & e no longer ac & \\
\hline Section & 1 - Instruments, players an & d voices & & & & & & & \\
\hline & & & & & & & & & \\
\hline $1.01 \mathrm{~A}$ & $\begin{array}{l}\text { il linguaggio musicale in } \\
\text { Zelenka, ... si puô recepire }\end{array}$ & $\begin{array}{l}\text { ndividuale di } \\
\text { nell' aria per }\end{array}$ & & alto & $\begin{array}{l}\text { con l'accompag } \\
\text { e archi }\end{array}$ & namento di flauti & http $: / / \mathrm{y}$ & $\begin{array}{l}\text { vww haendel.it/compositori/lavori/te } \\
\text { um_zelenka_cd.htm }\end{array}$ & $2016-05-31$ \\
\hline $1.01 \mathrm{~B}$ & Oliviri e Diana Tizzana, al vio & $\begin{array}{l}\text { olino, David } \\
\text { ombardi alla }\end{array}$ & & viola & e Giovanna Viv & aldi al violncello & http $/ / \mathrm{y}$ & $\begin{array}{l}\text { vww regione.piemonte it inotizie/piemonteinf } \\
\text { rchivio/territonio/archivio/2007/marzo htm }\end{array}$ & 2016-05-30 \\
\hline $1.01 \mathrm{C}$ & se una cantante è soprano,me & $\begin{array}{r}\text { zzosoprano } \\
\mathrm{a}\end{array}$ & & ontralto & $\begin{array}{l}\text { ? La mia insegna } \\
\text { cambia!Prima } \mathrm{m}\end{array}$ & $\begin{array}{l}\text { nnte ogni volta } \\
\text { i }\end{array}$ & $\begin{array}{l}\text { http://y } \\
\text { rispost } \\
\text { fonator }\end{array}$ & $\begin{array}{l}\text { vww liricamente it/esperto-musica-lirica- } \\
\text { e.asp?arg=fisiologia-apparato- } \\
\text { tio\&currentpage }=2\end{array}$ & 2016-05-30 \\
\hline $1.02 \mathrm{~A}$ & marimba e lo xilofono & $\begin{array}{l}\text { (in quanto } \\
\text { costituiti da }\end{array}$ & & Iamelle & $\begin{array}{l}\text { di legno disposte } \\
\text { su un supporto e }\end{array}$ & orizzontalmente & http $/ /$ fi & isicaondemusica unimore it/Percussioni html & 2016-06-01 \\
\hline $1.02 \mathrm{~B}$ & Ambiente accogliente e cald & do, il nostro & & bar & $\begin{array}{l}\text { propone le miglio } \\
\text { Trentino Alto }\end{array}$ & gri grappe del & http:/h & lotelpatrizia.tn.it/it- & 2016-05-30 \\
\hline Section & 2-Notation & & & & & & & & \\
\hline $2.01 \mathrm{~A}$ & tempi forti cioè il prim & $\begin{array}{l}\text { no e il terzo } \\
\text { imento della }\end{array}$ & & battuta & $\begin{array}{l}\text { e poi il secondo } \\
\text { noterâ che }\end{array}$ & e quarto. $\mathrm{Si}$ & http://y & $\begin{array}{l}\text { vww .cdpm.it/page asp?cat }=146 \& \text { desc=tutti- } \\
\text { yoglion-fare-jazz }\end{array}$ & $2016-05-30$ \\
\hline $2.01 \mathrm{~B}$ & tempi forti cioe 11 prim & $\begin{aligned} \text { no e il terzo } \\
\text { movimento }\end{aligned}$ & & misura & $\begin{array}{l}\text { e poi il secondo } \\
\text { noterà che }\end{array}$ & e quarto. $\mathrm{Si}$ & http $/ / \mathrm{y}$ & $\begin{array}{l}\text { vww cdpm.it } / \text { page asp?cat }=146 \& \text { desc }=\text { tutti- } \\
\text { yoglion-fare-jazz }\end{array}$ & $2016-05-30$ \\
\hline $2.01 \mathrm{C}$ & la legatura prolunga il suono & $\begin{array}{r}\text { anche dopo } \\
\text { la }\end{array}$ & & anghetta & , invadendo la mi & isura successiva. & http://y & $\begin{array}{l}\text { vww busonero it/2015/07-prolungamento- } \\
\text { ota/ }\end{array}$ & 2016-08-06 \\
\hline $2.01 \mathrm{D}$ & o per segnalare importanti ca & ambiamenti: & $p$ & a stanghetta & $\begin{array}{l}\text { di chiusura (due } \\
\text { cuil ta }\end{array}$ & linee verticali di & $\begin{array}{l}\text { http } / / \mathrm{d} \\
\text { grafico }\end{array}$ & $\begin{array}{l}\text { locplayer it/91682-Dal-suono-al-segno- } \\
\text { htm1 }\end{array}$ & 2016-08-06 \\
\hline
\end{tabular}




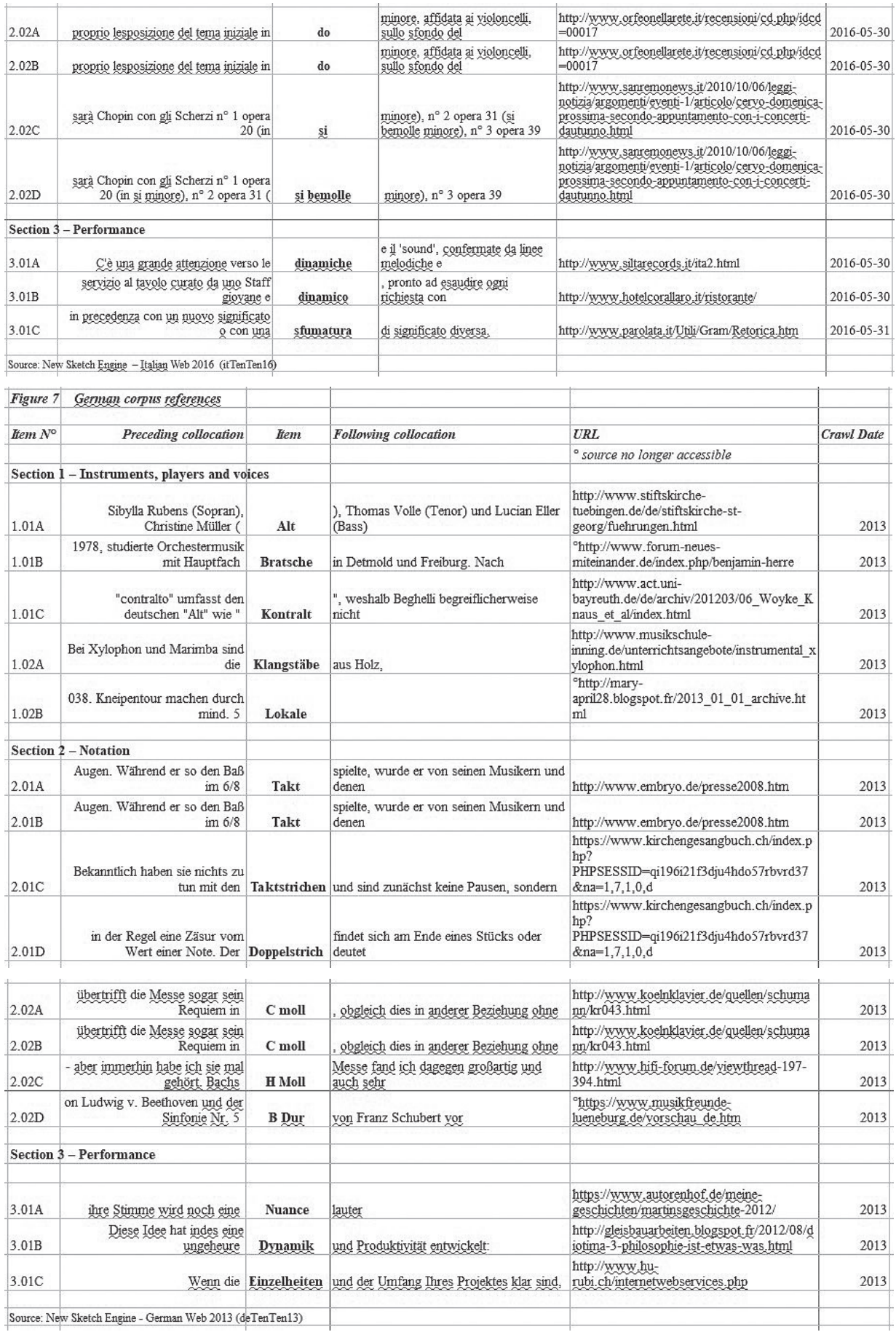




\begin{tabular}{|c|c|c|c|c|c|}
\hline Figure 8 & Russian corpus references & & & & \\
\hline \multirow[t]{2}{*}{ Item $N^{\circ}$} & Preceding collocation & Item & Following collocation & $U R L$ & Crawl Date \\
\hline & & & & - source no longer accessible & \\
\hline \multicolumn{6}{|c|}{ Section 1 - Instruments, players and voices } \\
\hline $1.01 \mathrm{~A}$ & сопрано , мемдо-сопрано, & альт & , тенор, бас & http $/ /$ www wikiznanie.ru & 2011 \\
\hline $1.01 \mathrm{~A}$ & soprano, mecco-soprano, & al't & , tenor, bas & /ru-wz/index.php/Orepa & \\
\hline $1.01 \mathrm{~A}$ & soprano, mezzo-soprano, & alto & , tenor, bass & & \\
\hline $1.01 \mathrm{~B}$ & Бетховен дуэт для & альта & $\begin{array}{l}\text { : виолончели й двух облигатнвх } \\
\text { очкариков. }\end{array}$ & http $/ /$ musicbravo.ru & 2011 \\
\hline $1.01 \mathrm{~B}$ & Bethoyen duèt dlia & al'ta & violončeli i dvuh obligatnyh očkarikoy. & /category/afisha/ & \\
\hline $1.01 \mathrm{~B}$ & Beethoven Duet for & Viola & and Cello and Two Eyeglasses Obligato & & \\
\hline $1.01 \mathrm{C}$ & Глубокое богатое & контральто & glubokoe bogatoe & http//www.mir11.ru & 2011 \\
\hline $1.01 \mathrm{C}$ & & contralto & kontral'to & imassa.php & \\
\hline $1.01 \mathrm{C}$ & Deep rich & contralto & & & \\
\hline $1.02 \mathrm{~A}$ & $\begin{array}{r}\text { высотой звучания. Представлgет собой } \\
\text { ряд деревяннгх }\end{array}$ & брусков & разной величины, & https $/ /$ ru. wikipedia.org & $2018-04-17$ \\
\hline $1.02 \mathrm{~A}$ & $\begin{array}{r}\text { vysotoj zyučanija. Predstavliaet soboj riad } \\
\text { derevjannyh }\end{array}$ & bruskoy & raznoj veličiny & & \\
\hline $1.02 \mathrm{~A}$ & High pitched. It is a series of wooden & bars & of different sizes, & & \\
\hline $1.02 \mathrm{~B}$ & кафе с живой музыкой вечером, & бар & с караоке, казино, & http $/ /$ santa-avia.ru/country & 2011 \\
\hline $1.02 \mathrm{~B}$ & kafe s živoj muzykoj večerom. & bar & s karaoke, kazino. & /yuzhnaya-koreya/resorts/ochedzhudo & \\
\hline $1.02 \mathrm{~B}$ & cafes with live music in the evening, & bars & with karaoke, casino, & hotels/hyatt-regency-cheju-5 & \\
\hline \multicolumn{6}{|c|}{ Section 2 - Notation } \\
\hline & & & & & \\
\hline $2.01 \mathrm{~A}$ & в арии, вкщючаношей фразу в пятналшать & тактов & $\begin{array}{l}\text { при сдержанном темпе (12/8), с двумя } \\
\text { периодами }\end{array}$ & http//belcanto.ru/norma.html & 2011 \\
\hline $2.01 \mathrm{~A}$ & v arii, vkliučajuŝej frazu v pjatnadcat' & taktov & $\begin{array}{l}\text { pri sderžannom tempe }(12 / 8) \text {, s drumja } \\
\text { periodami }\end{array}$ & & \\
\hline $2.01 \mathrm{~A}$ & $\begin{array}{r}\text { in the aria, which includes a phrase of } \\
\text { fifteen }\end{array}$ & bars & $\begin{array}{l}\text { at a moderato tempo (12/8), with two } \\
\text { periods }\end{array}$ & & \\
\hline & & & & & \\
\hline $2.01 \mathrm{~B}$ & в арии, включаюошей фразу в пятнадшать & тактов & $\begin{array}{l}\text { при сдержанном темпе (12/8), с двумя } \\
\text { периодами }\end{array}$ & http//belcanto ru/norma.html & 2011 \\
\hline $2.01 \mathrm{~B}$ & v arii, vkliučajuŝej frazu v pjatnadcat' & taktov & $\begin{array}{l}\text { pri sderžannom tempe (12/8), s dyumja } \\
\text { periodami }\end{array}$ & & \\
\hline $2.01 \mathrm{~B}$ & $\begin{array}{r}\text { in the aria, which includes a phrase of } \\
\text { fifteen }\end{array}$ & bars & $\begin{array}{l}\text { At a moderato tempo (12/8), with two } \\
\text { periods }\end{array}$ & & \\
\hline $2.01 \mathrm{C}$ & & Та́ктовая черта́ & (тактовой черте или черте доли такта) & httns//nu wikinedia org & $2018-04-19$ \\
\hline $2.01 \mathrm{C}$ & & $\begin{array}{l}\text { 1akToвag qepra } \\
\text { Táktoyaja čertá }\end{array}$ & Ispol'zuetsja dlja otdelenija taktov & https.//ru WWikjpedia.org & \\
\hline $2.01 \mathrm{C}$ & & bar line & Used for separating bars & & \\
\hline $2.01 \mathrm{D}$ & & $\underset{\text { черта }}{\text { Двойна́ }}$ & $\begin{array}{l}\text { Oтденяет друг от друга два фрагмента } \\
\text { произведения. }\end{array}$ & https $/ /$ ru. wikipedia.org & 2018-04-19 \\
\hline 2.01D & & dyoinájá táḱktovaia & $\begin{array}{l}\text { Qtdeliaet drug ot druga dya fragmenta } \\
\text { projzvedenija. }\end{array}$ & & \\
\hline $2.01 \mathrm{D}$ & & double bar & $\begin{array}{l}\text { Separates from each other two fragments } \\
\text { of the work. }\end{array}$ & & \\
\hline & & & & & \\
\hline $2.02 \mathrm{~A}$ & Соната №7 & до & Мนнор БЕТХOВЕНА & http $/ /$ www tatphilharmonia $r u / 1013$ & 2011 \\
\hline $2.02 \mathrm{~A}$ & Sonata №7 & do & minor BETHOVENA & & \\
\hline $2.02 \mathrm{~A}$ & Sonata №7 & C minor & BEETHOVEN & & \\
\hline $2.02 \mathrm{~B}$ & Соната №7 & до & MUH Op БETXOBEHA & http $/ /$ www tatphilharmonia.ru/1013 & 2011 \\
\hline $2.02 \mathrm{~B}$ & Sonata №7 & do & minor BETHOVENA & & \\
\hline $2.02 \mathrm{~B}$ & Sonata №7 & C minor & BEETHOVEN & & \\
\hline
\end{tabular}




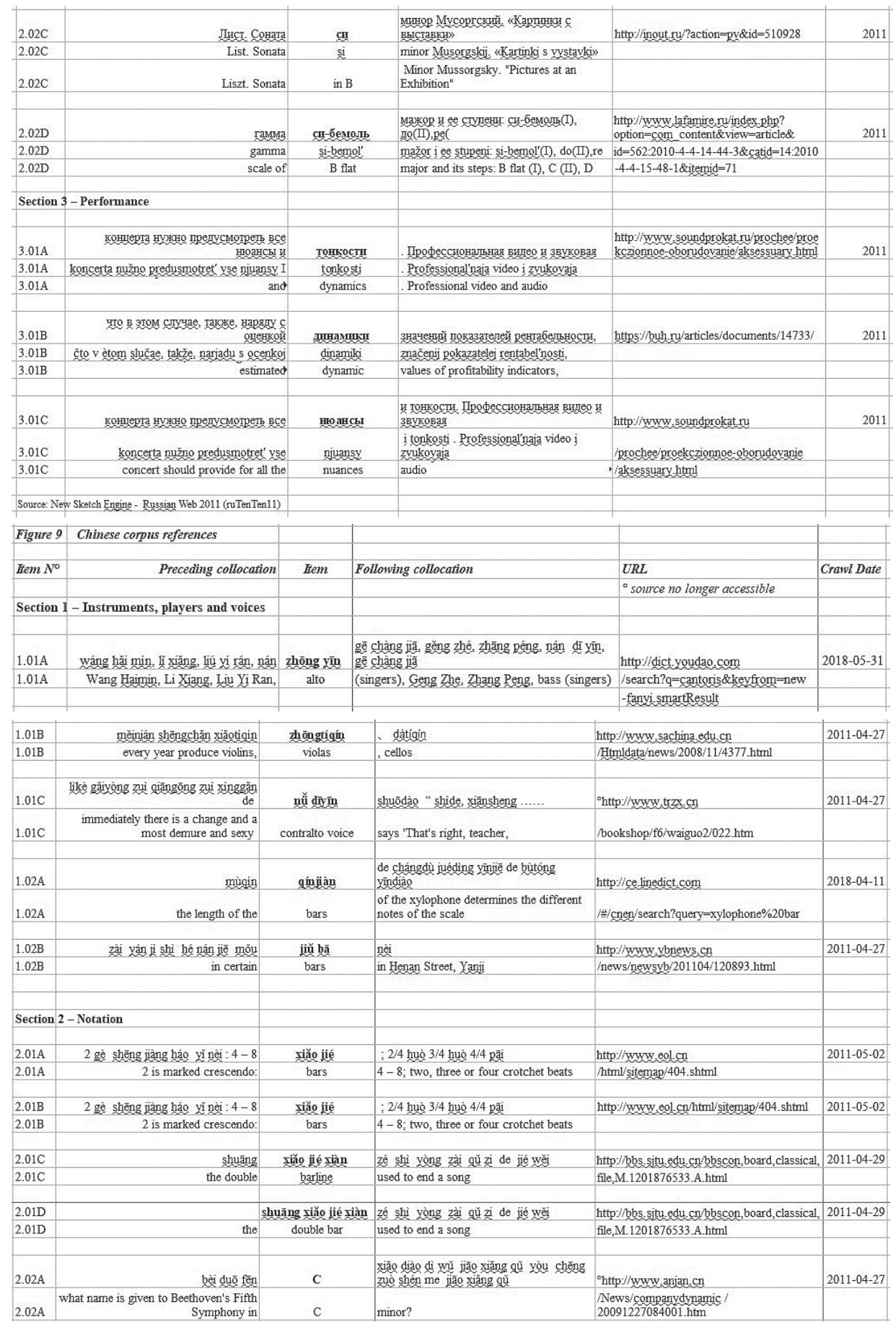




\begin{tabular}{|c|c|c|c|c|c|}
\hline $2.02 \mathrm{~B}$ & bèi duō fền & C & $\begin{array}{l}\text { xiăo diào dì wŭ jiāo xiăng gŭ yòu chēng } \\
\text { zuô shên me jiäo xiàng qú }\end{array}$ & ${ }^{\circ} \mathrm{http} / /$ www anjan cn & 2011-04-27 \\
\hline $2.02 \mathrm{~B}$ & $\begin{array}{r}\text { what name is given to Beethoven's Fifth } \\
\text { Symphony in }\end{array}$ & C & minor? & $\begin{array}{l}\text { /News/companydynamic / } \\
\text { 20091227084001.htm }\end{array}$ & \\
\hline $2.02 \mathrm{C}$ & $\begin{array}{r}\text { Franz Liszt ( } 1811-1886) \text { zuò jià } \\
\text { shēng ping zuó pìn }\end{array}$ & B & xiăo diào gāng gin zòu ming gŭ & ${ }^{\circ} \mathrm{http} / /$ www.insz.cn & 2011-04-27 \\
\hline $2.02 \mathrm{C}$ & $\begin{array}{r}\text { Franz Liszt ( } 1811-1886) \text { the } \\
\text { composition of his life: }\end{array}$ & B & minor piano sonata & /Portals//yinyuecheng/xiyangzhuming.htm & \\
\hline $2.02 \mathrm{D}$ & $\begin{array}{l}\text { shuāng huáng guăn hé dān huáng } \\
\text { guăn tóng shi zồ chü de }\end{array}$ & jiàng $B$ y⿳⺈.⿲㇒丨丶 & & http $/ /$ bbs.ustc. edu cn & 2011-04-29 \\
\hline $2.02 \mathrm{D}$ & $\begin{array}{r}\text { double reed instruments and clarinets } \\
\text { are played in }\end{array}$ & B flat & & $\begin{array}{l}\text { /cgi/bbscon? } \\
\text { bn=ClassicMusic\& fn=M319BFBD } 5 \text { \& }\end{array}$ & \\
\hline & & & & num $=499$ & \\
\hline \multicolumn{6}{|c|}{ Section 3 - Performance } \\
\hline & & & & & \\
\hline $3.01 \mathrm{~A}$ & & lìdù & shì zhì yònglì de giángdù & http $/ /$ wwww 360doc.com & $2018-05-20$ \\
\hline \multirow[t]{2}{*}{$3.01 \mathrm{~A}$} & & dynamics & refers to the degree of loudness & $/$ content $/ 16 / 0102 / 20 / 15266668$ & \\
\hline & & & & 524895136.shtml & \\
\hline & & & & & \\
\hline $3.01 \mathrm{~B}$ & & $\begin{array}{c}\text { shēeng qì bó } \\
\text { bó }\end{array}$ & de gănjué & ${ }^{\circ} \mathrm{http} / /$ yh, vip.oldkids,cn & 2011-04-27 \\
\hline $3.01 \mathrm{~B}$ & & dynamic & feeling & /new_page_48.htm & \\
\hline $3.01 \mathrm{C}$ & duo cì xún wèn huì bào nèi róng & ì̀ jié & wèn ti & 'http//www sdwfh org.cn/2011/0210/273. & 2011-04-27 \\
\hline $3.01 \mathrm{C}$ & quests have been made for a report with & details & of the content & & \\
\hline surce & Sketch Engine - Chinese Web 2011 (zhTenTen1 & 11, Stanford tag & ger) & & \\
\hline
\end{tabular}

\section{References:}

Clarke, H. A. 1896. Pronouncing Music Dictionary. Theodore Presser Co., Philadelphia https://archive.org/stream/pronouncingmusic00clar\#page/n7/mode/2up (accessed 5 July 2018).

Dumorest, Steven M. 2003. Building Choral Excellence: Teaching Sight-Singing in the Choral Rehearsal. Oxford University Press, London.

Gannaz, FrançoIs et AL (eds.). 2018 (latest online update). Le Littré. Dictionnaire de la langue française par É. Littré. https://www.littre.org (accessed 29 June 2018).

Humbley, John. 2010. Peut-on encore parler d'anglicisme? Léturgie, Arnaud (LDI). Lexique, normalisation, transgression. Cergy-Pontoise. Mes Mots Edition. Limay. 21-45.

Kennedy, Michael; Bourne, Joyce (eds.). 1996 (4 $4^{\text {th }}$ edition). The Concise Oxford Dictionary of Music. Oxford University Press. Oxford - New York.

Knospe, Sebastian; Onysko, Alexander; Goth, Maik. 2016. Crossing Languages to Play with Words: Multidisciplinary Perspectives. De Gruyter, Berlin.

Koessler, Maxime; Derocquigny, Jules. 1928. Les Faux Amis ou Les Trahisons du Vocabulaire Anglais. Vuibert, Paris.

Robert, PAul. 1950 (2 $2^{\text {nd }}$ edition 1985). Le Grand Robert de la langue française. Dictionnaires Le Robert. Paris. 
Sablayrolles, JeAn-François. 2010. Néologisme homonymique, néologisme polysémique et évolution de sens. Pour une restriction de la néologie sémantique. Alves, Ieda Maria (Org.). Neologia e neologismos en diferentes perspectivas. CNPq, Paulistana. 83-100 Scholes, Percy A. 1938 (10 $0^{\text {th }}$ edition 1970). The Oxford Companion to Music. Oxford University Press, London.

Siron, Jacques. 2002. Dictionnaire des Mots de la Musique. Outre Mesure. Paris.

Summers, Della (ed.). 1995 ( $3^{\text {rd }}$ edition). Longman Dictionary of Contemporary English. Longman, Harlow.

Ullman, Stephen. 1951. The Principles of Semantics. Blackwell. Oxford.

Zuckermann, Ghil'Ad. 2003. Language Contact and Lexical Enrichment in Israeli Hebrew. Palgrave Macmillan, Houndmills (Basingstoke).

\section{Online resources}

Artopium's Music Dictionary. https://musicterms.artopium.com/a/index.htm (accessed 4 July 2018).

Etymonline.com. https:/www.etymonline.com/word/bar (accessed 4 July 2018).

New Sketch Engine. https://www.sketchengine.eu/ (accessed many times February July 2018).

\section{Neki primjeri korpusnoga istraživanja potencijalno zbunjujućih polisema i lažnih prijatelja u glazbenome nazivlju usredotočeni na engleski i francuski s ekvivalentima iz pet drugih jezika}

\section{Sažetak}

Ovaj rad predstavlja tekuće istraživanje elemenata glazbene terminologije koji se čine sličnima na engleskom i francuskom jeziku, ali zapravo imaju drugačija značenja. Svi termini također su dani na talijanskom, njemačkom, španjolskom, ruskom i kineskom jeziku. Korištenje pojmova na svim jezicima koji su obuhvaćeni opravdano je uzorcima teksta preuzetima iz priznatih zbirki uključenih u "New Sketch Engine", ali kritički komentari bitno su ograničeni na engleski i francuski.

Studija je u osnovi sinkronizirana: prioritet se daje ispitivanju trenutačne upotrebe. Dijakronijska razmatranja također su uključena gdje je bilo moguće predložiti kako je došlo do semantičke varijacije između pojmova čija je etimološka izvedba slična.

Keywords: music terminology, false friends, English-French, ambiguity, semantic restriction Ključne riječi: glazbena terminologija, lažni prijatelji, engleski-francuski, dvosmislenost, semantičko ograničenje 